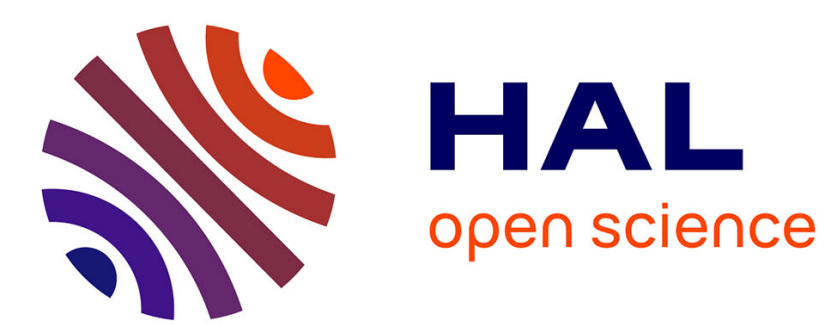

\title{
13. Émission de phonons par des quasi-particules dans des jonctions tunnel entre des supraconducteurs
}

\author{
H. Kinder, K. Lasmann, W. Eisenmerger
}

\section{To cite this version:}

H. Kinder, K. Lasmann, W. Eisenmerger. 13. Émission de phonons par des quasi-particules dans des jonctions tunnel entre des supraconducteurs. Revue de Physique Appliquée, 1970, 5 (6), pp.904-904. 10.1051/rphysap:0197000506090402 . jpa-00243483

\section{HAL Id: jpa-00243483 https://hal.science/jpa-00243483}

Submitted on 1 Jan 1970

HAL is a multi-disciplinary open access archive for the deposit and dissemination of scientific research documents, whether they are published or not. The documents may come from teaching and research institutions in France or abroad, or from public or private research centers.
L'archive ouverte pluridisciplinaire HAL, est destinée au dépôt et à la diffusion de documents scientifiques de niveau recherche, publiés ou non, émanant des établissements d'enseignement et de recherche français ou étrangers, des laboratoires publics ou privés. 


\title{
13. ÉMISSION DE PHONONS PAR DES QUASI-PARTICULES DANS DES JONCTIONS TUNNEL ENTRE DES SUPRACONDUCTEURS
}

\author{
H. KINDER, K. LASMANN et W. EISENMERGER
}

Université de Stuttgart

La variation de l'amplitude du signal avec le potentiel appliqué au générateur a été obtenue avec des jonctions Sn-I-Sn utilisées comme générateur et détecteur. Cette mesure fournit des informations sur les mécanismes de disparition des quasi-particules dans des supraconducteurs. La variation de la dérivée de l'amplitude du signal par rapport au courant de l'émetteur a été calculée en fonction du potenciel appliqué à l'émetteur. Pour ce faire nous avons utilisé la théorie de Tewordt sur le temps de vie des quasi-particules prenant en considération les différents processus possibles : relaxation et recombinaison. De plus nous avons tenu compte de la possibilité de réabsorption d'un phonon à partir d'une paire détruite, de la relaxation et de la recombinaison des quasi-particules dans l'émetteur. Nous obtenons ainsi une variation de la pente pour un potentiel appliqué au générateur de $6 \Delta / e$.

La forme générale de la courbe expérimentale est bien reproduite. Le saut du potentiel appliqué au générateur apparaît à $4 \Delta / e$ (commencement de la détection de la relaxation des phonons) ce qui donne une valeur à peu près $20 \%$ plus petite que celle prévue par la théorie. De même la variation de la pente à $6 \Delta / e$ concorde avec la théorie à quelque $20 \%$ près. On peut déduire que la réabsorption des phonons par l'intermédiaire d'une destruction de paires et les relaxations successives sont des mécanismes importants dans l'émission de phonons. 